\title{
Association between leukotriene receptor antagonist therapy and Churg-Strauss syndrome: an analysis of the FDA AERS database
}

\author{
S Bibby, ${ }^{1}$ B Healy, ${ }^{1}$ R Steele, ${ }^{1}{ }^{1,2}$ K Kumareswaran, ${ }^{1}$ H Nelson, ${ }^{3}$ R Beasley ${ }^{1,2}$
}

- Supplementary data are published online only at http:// thx.bmi.com/content/vol65/ issue2

${ }^{1}$ Medical Research Institute of New Zealand, Wellington, New Zealand ${ }^{2}$ Capital and Coast District Health Board Wellington, New Zealand ${ }^{3}$ National Jewish Health, Denver, Colorado, USA

\section{Correspondence to} Professor R Beasley, Medical Research Institute of New Zealand, P 0 Box 10055, Wellington 6143, New Zealand; richard.beasley@mrinz.ac.nz

Received 10 June 2009 Accepted 1 November 2009

\begin{abstract}
Background The possible role of leukotriene receptor antagonist (LTRA) therapy in the pathogenesis of ChurgStrauss syndrome (CSS) is uncertain. The aim was to examine the association between LTRA therapy and CSS in cases registered in the FDA Adverse Event Reporting System (AERS) database.
\end{abstract}

Methods All cases of suspected drug-induced CSS reported to the AERS database between November 1997 and April 2003 were reviewed. Subjects in whom LTRAs were the suspected medication and sufficient documentation existed to confirm the diagnosis of CSS were sequentially categorised into one of the following groups: (A) CSS before treatment initiation; (B) oral or inhaled corticosteroids reduced or stopped within 6 months of CSS onset; (C) possible prodromal phase of CSS at treatment initiation; (D) unstable asthma at treatment initiation; (E) stable asthma at treatment initiation.

Results There were 181 case reports of suspected druginduced CSS with sufficient documentation to confirm a diagnosis of CSS; in 163 (90\%) an LTRA was a suspect medication. In 140 of these 163 cases there was sufficient documentation to sequentially categorise the case into groups, with $13(9 \%)$ in $A, 27(19 \%)$ in $B, 11$ $(8 \%)$ in C, $28(20 \%)$ in D and $61(44 \%)$ in $\mathrm{E}$.

Conclusion LTRA therapy was a suspect medication in most confirmed cases of CSS reported in the AERS database. In the majority of cases treated with an LTRA, CSS could not be explained by either corticosteroid withdrawal or pre-existing CSS. These findings are informative in considering the potential associations between LTRA therapy and CSS.

\section{INTRODUCTION}

The possible role of leukotriene receptor antagonist (LTRA) therapy in the pathogenesis of Churg-Strauss syndrome (CSS) is uncertain, with conflicting views on whether the association may be causal or due to confounding by indication. ${ }^{1-4}$ A number of hypotheses have been proposed, including a causal relationship suggested by the temporal relationship between the introduction of LTRA therapy and development of CSS, and the reporting patterns to adverse drug reactions databases. Cases of CSS reported to the Committee on Safety of Medicines in the UK since the beginning of 1998 have mostly been associated with LTRAs. ${ }^{5}$ Likewise, a strong association between CSS and LTRAs has been noted in the US Food and Drug Administration (FDA) Adverse Event Reporting System (AERS) database. ${ }^{6}$ Another possibility is that LTRA therapy may allow a reduction in corticosteroid therapy leading to an unmasking of underlying CSS which had been suppressed by the corticosteroid therapy. ${ }^{7-11}$ A related hypothesis is that LTRA therapy may have been prescribed in response to severe asthma which represented an early prodromal phase of CSS, which then progressed to full expression of the disease. ${ }^{912}$ Previously it has been difficult to assess these hypotheses owing to the paucity of data, being limited primarily to either case reports or case series in which the method of case selection was not specified.

The clinical trial programme showing LTRAs to be effective in the treatment of asthma ${ }^{13}$ was not designed to detect the occurrence of rare serious adverse events such as CSS, which has a background incidence of approximately three per million in the general population. ${ }^{14}$ For any drug therapy, only once it is registered and widely used in clinical practice will rare adverse events or events in populations not examined in clinical trials become apparent and be reported by clinicians to adverse drug reactions databases. We have analysed cases of CSS reported to the FDA AERS database, focusing on an investigation of (1) the percentage of cases associated with the use of LTRA therapy; (2) patterns of disease and their relation to treatment, in particular corticosteroid withdrawal and possible pre-existing disease; and (3) characteristics of the disease present in this population.

\section{METHODS}

All cases in the FDA AERS database from November 1997 (when the term allergic granulomatous angiitis was introduced) to April 2003 of suspected drug-induced CSS were provided by the FDA under the provisions of the Freedom of Information Act (see online supplement). In some of the case reports provided, key dates relating to disease therapy and CSS onset had been variably blacked out by the FDA; for this reason, we also accessed cases previously provided by the FDA for a related research project ${ }^{6}$ in which no details were blacked out.

Information from the MedWatch report form was entered onto a questionnaire in Microsoft Access. Objective data were entered by one investigator ( $\mathrm{SB}$ or $\mathrm{BH}$ ), while subjective data were entered independently by both these investigators, with any differences adjudicated by discussion with a third investigator (RS or RB).

\section{Exclusion criteria for case reports}

Cases were excluded sequentially by the first applicable category as outlined in box 1 . Sequential application of the exclusion criteria resulted in the 
inclusion of cases with a confirmed diagnosis of CSS in which an LTRA agent was reported as the suspect medication and which had been prescribed within 6 months of CSS onset. These cases were included in further analyses.

\section{Analysis of LTRA cases with confirmed diagnosis of CSS}

The demographic characteristics and clinical features of the cases were recorded, including organ involvement, presence of antineutrophil cytoplasmic antibodies (ANCA) and histological results from biopsy or post-mortem examination. The number of American College of Rheumatology (ACR) criteria reached before beginning LTRA and during the illness were also recorded. Where documented, we calculated the time period from initiation of LTRA therapy to onset of CSS, defined as the date when the case was first documented to have met one of the three diagnostic criteria to confirm the diagnosis of CSS, as stated in box 1. In cases where the onset of CSS preceded LTRA commencement, the time to onset was not calculated.

\section{Patterns of LTRA use, disease presentation and corticosteroid use}

Criteria were used to stratify cases according to patterns of LTRA use in relation to disease presentation and corticoste-

\section{Box 1 Exclusion criteria sequentially applied to the case} reports

1. Duplicate reports for the same case: as identified by unique registration numbers, age, sex, date of onset of Churg-Strauss syndrome (CSS), treatment start dates and any other unique identifying feature. Such reports were combined and recorded as a single case.

2. Report from a non-health professional: those not reported by a health professional (such as a physician, pharmacist, or nurse) were excluded (ie, reports from the patient, family member or lawyer only).

3. Diagnosis not CSS: where the recorded diagnosis was not CSS (ie, wrongly filed), or if at the conclusion of the report the reporting physician considered an alternative diagnosis more likely.

4. Other reason for exclusion: including conference proceedings, case series (not providing data on individual patients), illegible reports and those which were later withdrawn by the reporter.

5. Insufficient documentation to confirm the diagnosis of CSS: where cases did not meet one of the following three criteria (see online supplement):

- At least four of the six American College of Rheumatology (ACR) classification criteria for CSS. ${ }^{15}$

- History of asthma AND eosinophilia AND histology consistent with CSS on biopsy.

- Post-mortem findings confirming the diagnosis of CSS.

6. Suspect therapy(ies) asthma medication(s) other than a leukotriene receptor antagonist (LTRA): where the suspect medication(s) listed included an asthma treatment other than an LTRA agent (regardless of concurrent LTRA use if not reported as a suspect medication).

7. Suspect therapy(ies) not asthma medication(s): where the suspect medication(s) was/were not used in the treatment of asthma (regardless of concurrent LTRA use if not reported as a suspect medication).

8. LTRA therapy ceased $>6$ months before onset of CSS. roid use, in line with current hypotheses proposed to explain the association between LTRA therapy and CSS, as shown in table 1

\section{RESULTS}

There were 1274 case reports of suspected drug-induced CSS in the AERS database. The date of CSS onset ranged from 18 December 1996 to 5 April 2003. The sequential application of the exclusion criteria is shown in figure 1. There were 181 cases of suspected drug-induced CSS reported by a health professional in which there was sufficient documentation to confirm the diagnosis of CSS.

Among these 181 cases there were 12 in whom an asthma medication(s) other than an LTRA was reported as the suspect medication (fluticasone propionate, $\mathrm{n}=6$; fluticasone propionate and salmeterol, $\mathrm{n}=4$; budesonide, $\mathrm{n}=1$; zileuton, $\mathrm{n}=1$ ), a further 5 in whom a non-asthma medication(s) was reported as the suspect medication and in 1 case the LTRA agent was stopped $>6$ months before the onset of CSS. There were 163/ $181(90 \%)$ cases of confirmed CSS in which an LTRA was reported as at least one of the suspect medications, four of which listed a non-LTRA medication as another suspect medication.

\section{Analysis of LTRA cases with a confirmed diagnosis of CSS}

Among the 163 cases, a confirmed diagnosis of CSS was made by the documentation of $\geq 4$ ACR criteria in 145 (89\%), by documentation of a history of asthma, eosinophilia and histology consistent with CSS in a further $17(10.4 \%)$ and by post-mortem findings of CSS in 1 additional case. There were six reported deaths among the 163 cases. The age range was 7-80years (median 51 years; in two cases age not specified), with a female: male ratio of 1.1:1. The time from starting LTRA therapy to disease onset ranged from 3 to 1340 days (figure 2). The LTRA medications reported included montelukast $(n=114)$, zafirlukast $(\mathrm{n}=43)$, montelukast and zafirlukast $(\mathrm{n}=5)$, montelukast and pranlukast $(n=1)$

Figure 3 shows the percentage of patients with documentation of the clinical features associated with CSS. A biopsy was taken in 128 of the 163 cases (79\%), with characteristic histological features of CSS reported in 112 (88\%). ANCA were measured in 89 patients, 37 of whom were positive $(42 \%)$. Five patients were both pANCA and cANCA positive, while 28 were pANCA and/or myeloperoxidase (MPO) positive only, 3 were cANCA positive only and 1 was not specified.

For the cases in whom an LTRA was reported as a suspect medication and in whom the date of onset of CSS was available, there were 19, 22, 26, 21, 14 and 6 cases reported yearly from 1997 to 2002 , respectively.

\section{Patterns of LTRA use, disease presentation and corticosteroid use}

In 140 of the 163 cases there was sufficient documentation to sequentially categorise the patterns of disease presentation and corticosteroid use (table 2, figure 4).

Group A included 13/140 (9.3\%) patients, of whom 5 had a previous diagnosis of CSS and 8 reached $\geq 4 / 6$ ACR criteria before initiation of LTRA therapy. Four would otherwise have met the criteria for Group B but are not included in Group B owing to sequential allocation of cases.

Group B included 27/140 (19.3\%) patients, of whom there were 21 with withdrawal or reduction in dose of OCS, 5 of ICS and 1 in whom the route of corticosteroid therapy was not specified. 


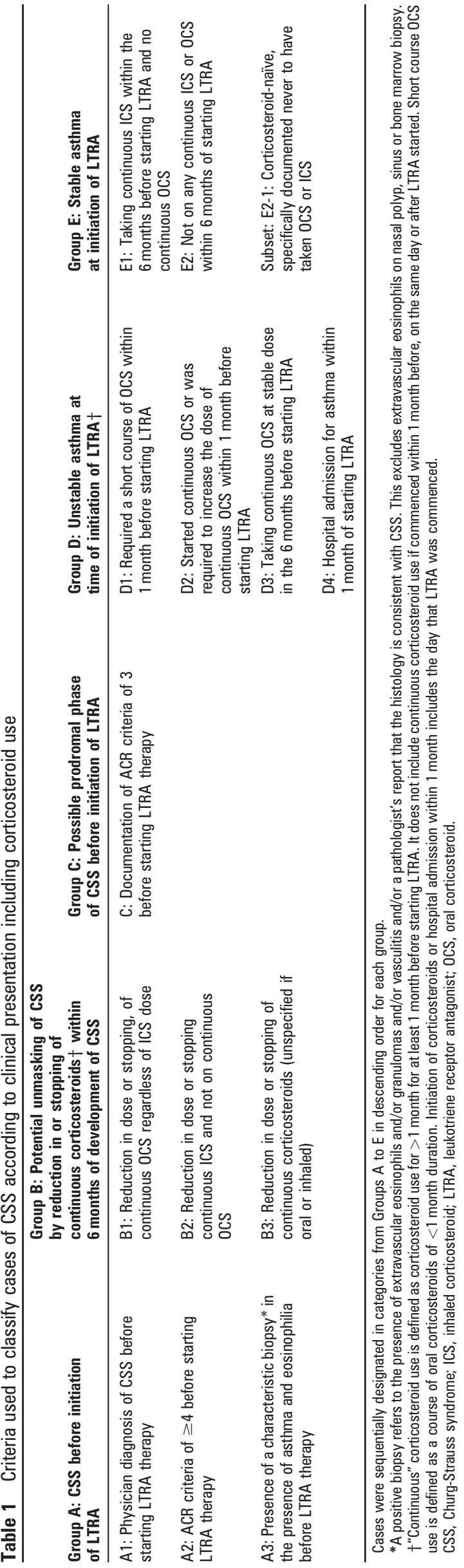

Group C included 11/140 (7.9\%) patients.

Group D included 28/140 (20\%) patients. When these cases were sequentially allocated to the subgroups, 18 had OCS started in the month before initiation of LTRA therapy (17 a short course and 1 continuous); 9 were on continuous OCS for at least a month before starting LTRA; and 1 had no OCS but a hospital admission for asthma in the month before starting LTRA therapy.

Group E included 61/140 (43.6\%) patients, of whom 52 were on regular ICS (with no continuous OCS in the 6 months before starting LTRA, and no short courses of OCS in the 1 month before starting LTRA); 9 did not take regular OCS or ICS, 5 of whom were documented to have never received OCS or ICS therapy.

\section{DISCUSSION}

To our knowledge, this review of the FDA AERS database represents the largest case series of CSS. It identified that, in $90 \%$ of the cases of confirmed CSS, an LTRA agent was a suspect medication. Of the cases of CSS in which an LTRA was a suspect medication and there was adequate documentation to classify the case, $36 \%$ had pre-existing CSS, had reduced or stopped oral or inhaled corticosteroid therapy, or had possible prodromal CSS at the time of initiation of LTRA therapy.

Before discussing these findings we will consider the methodological issues potentially limiting this analysis. First, some reports had inadequate details documented, others had conflicting information from different sources and, in others, key dates were blacked out by the FDA. As a result, less than half of the case reports had adequate documentation to enable confirmation of the diagnosis of CSS. Furthermore, among the cases of CSS associated with LTRA use, categorisation according to clinical presentation including corticosteroid use was difficult, with adjudication by a third investigator required in $20 \%$ of the 163 cases. Thus, one of our recommendations is that a more comprehensive and effective method of data documentation and investigation is required in patients who are reported to have suffered a serious adverse event in association with a suspected drug.

The next issue is whether there may have been preferential reporting of LTRA therapy because it was a novel treatment in asthma. In $90 \%$ of confirmed cases of CSS an LTRA was a suspect medication, compared with long-acting $\beta$-agonist (LABA) drugs which were reported in $<3 \%$ despite being introduced during a similar period and recommended for use in moderate to severe asthma. This contrasts with a recent European case-control study in which $19 \%$ of patients with CSS were receiving treatment with an LTRA compared with $63 \%$ on LABAs in the 3 months before the CSS diagnosis. ${ }^{16}$ Similarly, a US-based case-control study reported that $13 \%$ of patients with CSS were prescribed LTRA therapy in the $2-6$ months before the CSS diagnosis. ${ }^{17}$ Based on these data, it is likely that the high percentage of cases of CSS in which LTRAs were reported as the suspect medication in the FDA database is due in part to reporting bias, and that many cases of CSS occur without a medication being suspected and therefore are not reported. In our analysis there was no trend of an increased number of CSS events associated with LTRA therapy throughout the initial 6-year period of the database, despite LTRA therapy becoming a widely prescribed medication and the association with CSS being reported in the medical literature. This suggests that increasing awareness of the potential association between CSS and LTRA therapy did 
Figure 1 Allocation of cases with sequential application of the exclusion criteria as documented in the text. CSS, Churg-Strauss syndrome; LTRA, leukotriene receptor antagonist.

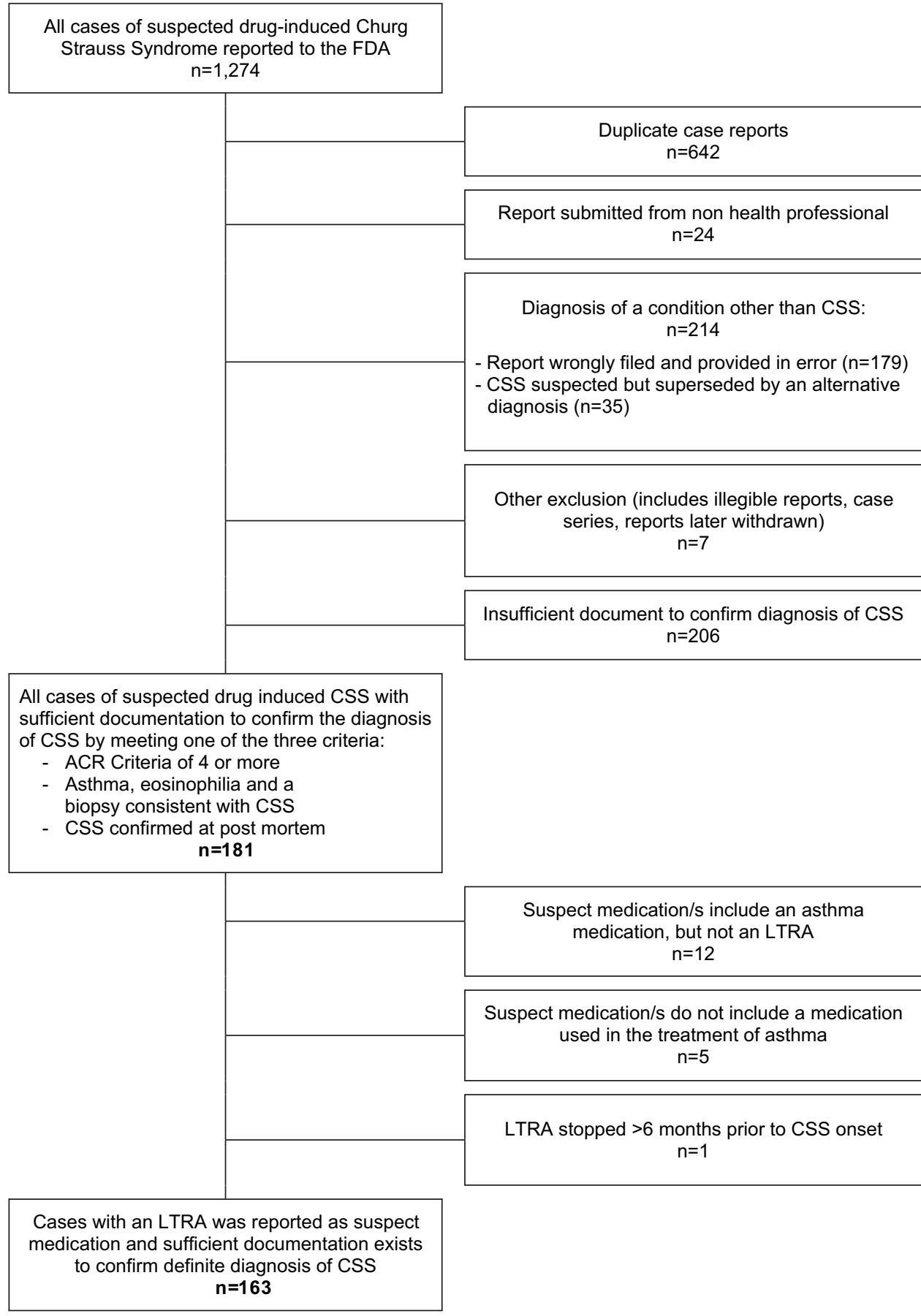

Figure 2 Time to onset of Churg-Strauss Syndrome (CSS) from start date of leukotriene receptor antagonist (LTRA) therapy in 108 of 163 cases of confirmed CSS in which an LTRA drug was reported as a suspect medication and in which there was sufficient documentation to determine this variable. In 13 cases the onset of CSS preceded the initiation of LTRA therapy (not shown).

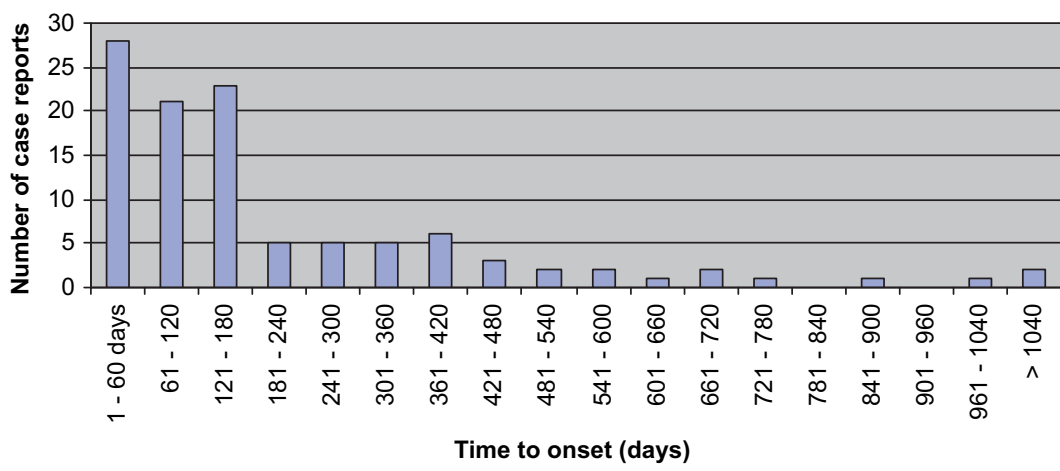


Figure 3 Clinical manifestations reported in the 163 cases of confirmed Churg-Strauss syndrome in which a leukotriene receptor antagonist drug was reported as a suspect medication.

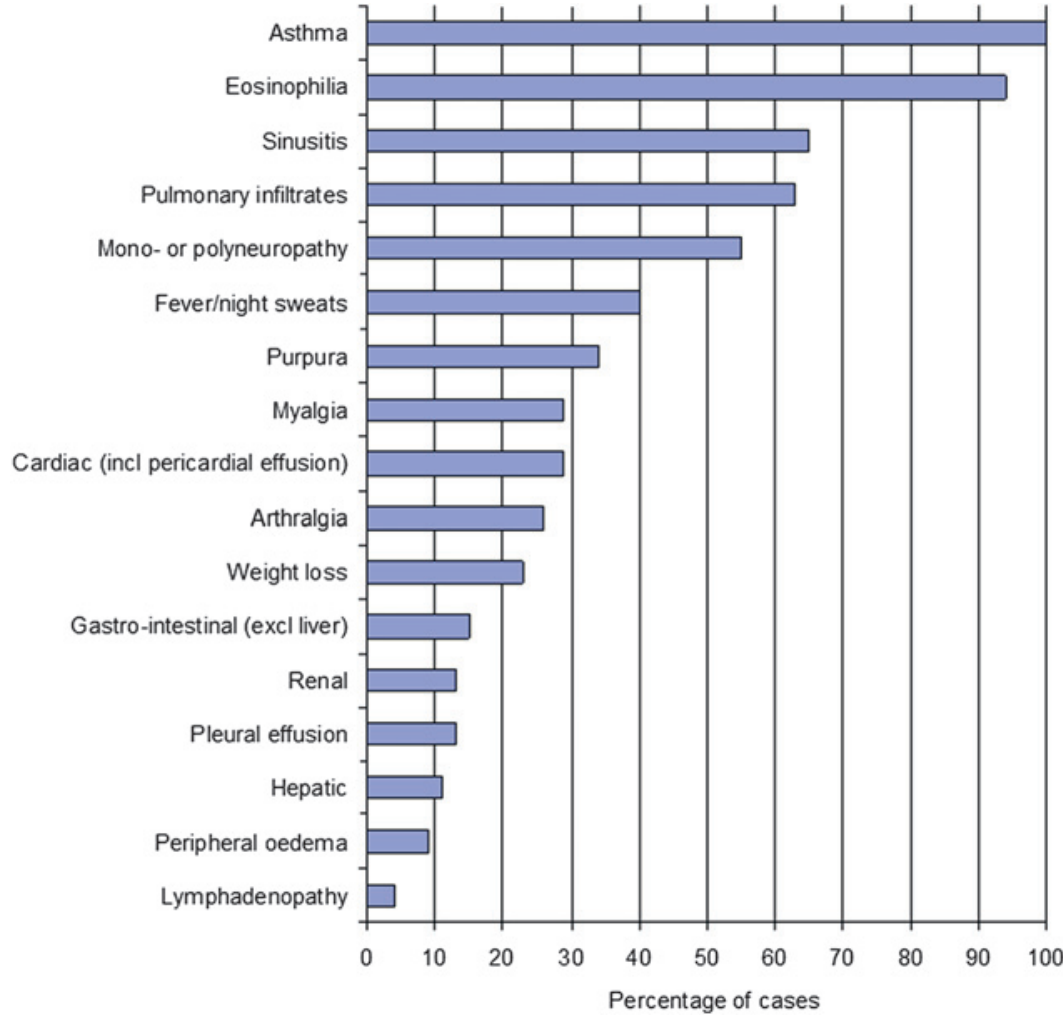

not lead to a greater propensity to report cases to the FDA database.

Despite these limitations, our study found that clinical manifestations of CSS were similar to those reported previously, with asthma, eosinophilia and multisystem involvement including sinusitis, mononeuritis multiplex and polyneuropathy, vascular skin lesions, pulmonary, musculoskeletal, gastrointestinal and cardiac disease. About $40 \%$ of cases in whom ANCA was measured were positive. As a result, it appears that the spectrum of multisystem involvement and ANCA status was similar to that observed in other case series of CSS. ${ }^{18-20}$

Based on the clinical situations hypothesised to account for the observed association between LTRA therapy and CSS, we subsequently categorised cases from the least to the greatest probability that the association was causal. The first situation, where patients developed CSS before initiation of LTRA therapy, was evident in $9 \%$ of cases.

The next situation, that LTRA therapy leads to an improvement in asthma control-allowing tapering or stopping of corticosteroids and thereby expression of CSS which was previously partially suppressed by corticosteroid therapy ${ }^{7-10}$ - was observed in $19 \%$ of cases. While it is debatable whether a decrease in inhaled corticosteroid dose would be sufficient to precipitate systemic vasculitis, it was included within this category as previously proposed. ${ }^{12} 21$ In contrast to our results, Nathani et al reported that $37 \%$ of cases of CSS associated with LTRA therapy published in the medical literature occurred in relation to corticosteroid withdrawal. ${ }^{2}$ This may indicate preferential reporting of corticosteroid tapering in the medical literature or under-reporting of such cases to the FDA.

The third situation proposes that LTRA therapy is prescribed in response to the initial manifestations of a prodromal phase of CSS and that subsequent progression to eosinophilic tissue infiltration and systemic vasculitis occurred as a result of natural disease progression. ${ }^{9} 12$ In $8 \%$ of cases an LTRA was prescribed in the setting of three ACR criteria of CSS, with the subsequent development of confirmed CSS. In these cases it was not possible to determine whether the association was causal or simply coincidental.

Thus, there was in total $36 \%$ of cases in whom the previously proposed hypothesis of pre-existing CSS, a reduction or stopping of oral or inhaled corticosteroid therapy or a possible prodromal phase of CSS were present. Conversely, in 64\% of patients, CSS could not be attributed to these hypotheses. Among these remaining cases, approximately one-third had poorly controlled asthma when LTRA therapy was started

Table 2 Number (and percentage) of cases designated sequentially in each category

\begin{tabular}{|c|c|c|}
\hline Group & Number & Percentage \\
\hline Group A: CSS before treatment initiation & 13 & 9.3 \\
\hline $\begin{array}{l}\text { Group B: Oral or inhaled corticosteroids } \\
\text { reduced or stopped within } 6 \text { months } \\
\text { of CSS onset }\end{array}$ & $\begin{array}{l}27[\mathrm{~B} 1=21, \mathrm{~B} 2=5, \\
\mathrm{B} 3=1]\end{array}$ & 19.3 \\
\hline $\begin{array}{l}\text { Group C: Possible prodromal early phase } \\
\text { of CSS at treatment initiation }\end{array}$ & 11 & 7.9 \\
\hline $\begin{array}{l}\text { Group D: Unstable asthma at treatment } \\
\text { initiation }\end{array}$ & $\begin{array}{l}28[D 1=17, D 2=1 \\
D 3=9, D 4=1]\end{array}$ & 20.0 \\
\hline $\begin{array}{l}\text { Group E: Stable asthma at treatment } \\
\text { initiation }\end{array}$ & $\begin{array}{l}61[E 1=52, E 2=9, \\
E 2-1=5]\end{array}$ & 43.6 \\
\hline
\end{tabular}

B1: Reduction in dose or stopping of continuous OCS, regardless of ICS dose. B2: Reduction in dose or stopping of continuous ICS, and not on continuous OCS B3: Reduction in dose or stopping of continuous corticosteroid (unspecified if oral or inhaled). D1: Required a short course of OCS within 1 month before starting LTRA.

D2: Started continuous OCS or was required to increase the dose of continuous OCS within 1 month before starting LTRA.

D3: Taking continuous OCS at a stable dose in the 6 months before starting LTRA. D4: Hospital admission for asthma within 1 month of starting LTRA.

E1: Taking continuous ICS within the 6 months before starting LTRA and no continuous OCS. E2: Not on any continuous ICS or OCS within 6 months of starting LTRA.

E2-1: Corticosteroid-naïve, specifically documented never to have taken OCS or ICS. CSS, Churg-Strauss syndrome; ICS, inhaled corticosteroid; LTRA, leukotriene receptor antagonist; OCS, oral corticosteroid. 


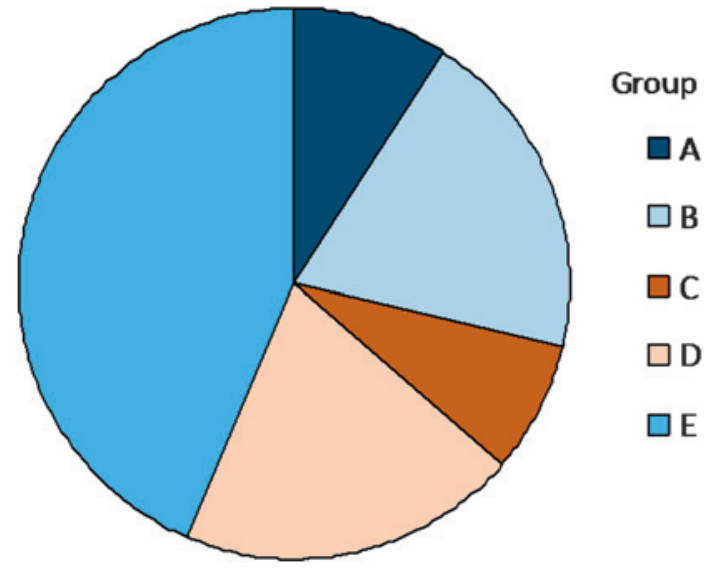

Figure 4 Percentage of cases designated sequentially in each category defined by patterns of disease and their relation to treatment. The figure includes the 140 cases in whom a leukotriene receptor antagonist was reported as a suspect medication and where sufficient documentation existed to both confirm a diagnosis of Churg-Strauss syndrome and sequentially to categorise into groups $A, B, C, D$ or $E$.

while two-thirds had stable asthma. It could be proposed that unstable asthma represents the earliest clinical manifestation of CSS, but this has not been done as oral corticosteroid use for exacerbations of asthma is common and the likelihood that it represents early CSS is extremely low. In those with stable asthma, causation was more likely as no other cause was identified as a precipitant of CSS. This group included five cases who had never received oral or inhaled corticosteroid therapy.

These findings are complemented by case-control studies of CSS and medication use. In the European study ${ }^{16}$ there was a significant risk of developing CSS with montelukast (OR 6.7, 95\% CI 1.3 to 34.1 ) but not LABA (OR 2.9, 95\% CI 0.6 to 13.3) or inhaled corticosteroids (OR 1.0, 95\% CI 0.2 to 4.8), although the wide confidence intervals led to some uncertainty in the interpretation of these findings. In the US study, ${ }^{17}$ a strong association between LTRA use and CSS was reported (OR 4.0, 95\% CI 1.49 to 10.6), but in the multivariate analysis controlling for other asthma drug use, no significant association was observed (OR $1.32,95 \%$ CI 0.44 to 3.96 ).

The pathophysiological mechanisms by which CSS may develop as a result of LTRA therapy are not clear. Indeed, the mechanisms underlying all cases of CSS are not well understood. ${ }^{22}$ It has been postulated that the use of LTRAs may lead to imbalances between blockade of the actions of cysteinyl-leukotriene (cysLT) (LTC4, LTD4, and LTE4) on the cysteinyl-leukotriene receptor 1 (cysLT1) and the actions of other mediators such as leukotriene B4 (LTB4) and 5-oxo-6,8,11,14-eicosatetraenoic acid. ${ }^{43-26}$ LTB4 has been shown to have biological effects on proinflammatory cells including neutrophils, eosinophils, macrophages, basophils, mast cell precursors and lymphocytes. ${ }^{27-31}$ The unopposed actions of cysLT through the cysteinyl-leukotriene receptor 2 (cysLT2) or through other uncharacterised receptors cannot be discounted. ${ }^{32}$ More information is required to support any postulated pathophysiological mechanisms underlying the association of LTRAs and CSS.

In conclusion, LTRA therapy was a suspect medication in $90 \%$ of confirmed cases of CSS in the FDA AERS database. Among the cases of CSS associated with LTRA use in which there was adequate documentation, about two-thirds were not related to a reduction in oral or inhaled corticosteroid therapy, pre-existing or possible prodromal CSS. We recognise that it is not possible to determine in individual cases whether the association between CSS and LTRA therapy is causal, coincidental or directly related to other patterns of disease presentation or medication use. However, we cautiously suggest that, in a majority of cases, LTRA therapy may have a role in the pathogenesis of this disorder. We consider that these findings justify more intensive epidemiological study of the role of LTRA therapy in the pathogenesis of CSS.

Funding Asthma and Respiratory Foundation of NZ, P 0 Box 1459, Wellington, New Zealand; Bowen Trust Board, P O Box 22117, Khandallah, Wellington, New Zealand.

Competing interests RB has received research grants and fees for consulting and/or speaking from AstraZeneca, GlaxoSmithKline and Novartis. HN has received research grants from Schering-Plough, Novartis, Genentech, Ception and AstraZeneca, fees for consulting from Genentech/Novartis, Abbot Laboratories, MediciNova, AstraZeneca, Amgen, GlaxoSmithKline, Schering-Plough, Dyson and Sepracor and is a member of the GlaxoSmithKline Speakers' Bureau. RB and HN were co-authors in the previous publication of the association of asthma medication with CSS based on the FDA AERS database $^{6}$ which was supported by GlaxoSmithKline. No pharmaceutical company had any involvement in the planning, design, analysis or interpretation of the current study. All other authors declare that they have no conflict of interest.

Provenance and peer review Not commissioned; externally peer reviewed.

\section{REFERENCES}

1. Beasley R, Bibby S, Weatherall M. Leukotriene receptor antagonist therapy and Churg-Strauss syndrome: culprit or innocent bystander? Thorax 2008:63:847-9.

2. Nathani N, Little MA, Kunst $\mathrm{H}$, et al. Churg-Strauss syndrome and leukotriene antagonist use: a respiratory perspective. Thorax 2008;63:883-8.

3. Stoloff S, Stempel D, Wechsler M, et al. Churg-Strauss syndrome: is there an association with leukotriene modifiers? Chest 2000;118:1515-6.

4. McDanel D, Muller BA. The linkage between Churg-Strauss syndrome and leukotriene receptor antagonists: fact or fiction? Ther Clin Risk Manag 2005;1:125-40.

5. Committee on Safety of Medicines/Medicines Control Agency. Leukotriene receptor antagonists update on adverse reaction profiles. Curr Probl Pharmacovigilance 1999;25:14.

6. DuMouchel W, Smith ET, Beasley R, et al. Association of asthma therapy and ChurgStrauss syndrome: an analysis of post-marketing surveillance data. Clin Ther 2004;26:1092-104.

7. Wechsler ME, Garpestad E, Flier SR, et al. Pulmonary infiltrates, eosinophilia, and cardiomyopathy following corticosteroid withdrawal in patients with asthma receiving zafirlukast. JAMA 1998;279:455-7.

8. Wechsler ME, Pauwels R, Drazen JM. Leukotriene modifiers and Churg-Strauss syndrome: adverse effect or response to corticosteroid withdrawal? Drug Saf 1999;21:241-51.

9. Lilly CM, Churg A, Lazarovich M, et al. Asthma therapies and Churg-Strauss syndrome. J Allergy Clin Immunol 2002;109:S1-20.

10. Mukhopadhyay A, Stanley NN. Churg-Strauss syndrome associated with montelukast. Postgrad Med J 2001;77:390-1.

11. Churg A, Brallas M, Cronin SR, et al. Formes frustes of Churg-Strauss syndrome. Chest 1995; 108:320-3.

12. Weller $\mathbf{P}$, Plaut $M$, Taggart $\mathrm{V}$, et al. The relationship of asthma therapy and ChurgStrauss syndrome: NIH workshop and summary report. J Allergy Clin Immunol 2001; 108:175-83.

13. O'Byrne PM, Gauvreau GM, Murphy DM. Efficacy of leukotriene receptor antagonists and synthesis inhibitors in asthma. J Allergy Clin Immunol 2009;124:397-403.

14. Noth I, Strek M, Leff A. Churg-Strauss syndrome. Lancet 2003;361:587-94

15. Masi A, Hunder G, Lie J, et al. The American College of Rheumatology 1990 criteria for the classification of Churg-Strauss syndrome (allergic granulomatosis and angiitis). Arthritis Rheum 1990;33:1094-100.

16. Hauser T, Mahr A, Metzler C, et al. The leucotriene receptor antagonist montelukast and the risk of Churg-Strauss syndrome: a case-crossover study. Thorax 2008:63:677-82

17. Harrold L, Patterson M, Andrade S, et al. Asthma drug use and the development of Churg-Strauss syndrome (CSS). Pharmacoepidemiol Drug Saf 2007;16:620-6.

18. Keogh K, Specks U. Churg-Strauss syndrome: clinical presentation, antineutrophil cytoplasmic antibodies, and leukotriene receptor antagonists. Am J Med 2003;115:284-90.

19. Sable-Fourtassou R, Cohen P, Mahr A, et al and the French Vasculitis Study Group. Antineutrophil cytoplasmic antibodies and the Churg-Strauss syndrome. Ann Intern Med 2005;143:632-8.

20. Solans R, Bosch J, Perez-Bocanegra C, et al. Churg-Strauss syndrome: outcome and long-term follow-up of 32 patients. Rheumatology 2001;40:763-71.

21. Hashimoto $\mathbf{M}$, Fujishima T, Tanaka $\mathrm{H}$, et al. Churg-Strauss syndrome after reduction of inhaled corticosteroid in a patient treated with pranlukast for asthma. Intern Med 2001:40:432-4. 
22. Tsurikisawa N, Saito $H$, Tsuburai $T$, et al. Differences in regulatory T cells between Churg-Strauss syndrome and chronic eosinophilic pneumonia with asthma. J Allergy Clin Immunol 2008;122:610-6.

23. Conen D, Leuppi J, Bubendorf L, et al. Montelukast and Churg-Strauss syndrome. Swiss Med Wkly 2004;134:377-80.

24. Guilpain $\mathbf{P}$, Viallard JF, Lagarde $\mathbf{P}$, et al. Churg-Strauss syndrome in two patients receiving montelukast. Rheumatology (Oxford) 2002;41:535-9.

25. Solans R, Bosch JA, Selva A, et al. Montelukast and Churg-Strauss syndrome. Thorax 2002;57:183-5.

26. Stirling RG, Chung KF. Leukotriene antagonists and Churg-Strauss syndrome: the smoking gun. Thorax 1999:54:865-6.

27. Dahlen SE. Treatment of asthma with antileukotrienes: first line or last resort therapy? Eur J Pharmacol 2006;533:40-56.
28. likura M, Suzukawa M, Yamaguchi $M$, et al. 5-Lipoxygenase products regulate basophil functions: 5-0xo-ETE elicits migration, and leukotriene B4 induces degranulation. J Allergy Clin Immunol 2005;116:578-85.

29. Rubin P, Mollison KW. Pharmacotherapy of diseases mediated by 5-lipoxygenase pathway eicosanoids. Prostaglandins Other Lipid Mediat 2007:83:188-97.

30. Evans JF, Ferguson AD, Mosley RT, et al. What's all the FLAP about?: 5-lipoxygenase-activating protein inhibitors for inflammatory diseases. Trends Pharmacol Sci 2008:29:72-8.

31. Harizi H, Corcuff JB, Gualde N. Arachidonic-acid-derived eicosanoids: roles in biology and immunopathology. Trends Mol Med 2008;14:461-9.

32. Lee TH, Woszczek G, Farooque SP. Leukotriene E4: perspective on the forgotten mediator. J Allergy Clin Immunol 2009;124:417-21.

\section{Pulmonary puzzle}

\section{A 69-year-old smoker with mediastinal and hilar lymphadenopathy}

\section{CLINICAL PRESENTATION}

A 69-year-old male heavy smoker was referred to our service with 6 months minimal productive cough and 2 weeks pleuritic central chest pain, with no weight loss or constitutional symptoms. There was no relevant previous medical history. Clinical examination demonstrated no abnormalities. CT chest revealed extensive mediastinal and right hilar lymphadenopathy with a conglomerate subcarinal lymph node of $7 \times 3.9 \mathrm{~cm}$, with no parenchymal lung abnormalities identified.

Whole-body ${ }^{18}$ fluorodeoxyglucose (FDG) positron emission tomography revealed increased nodal uptake throughout the mediastinum (figure 1) as well as in the right supraclavicular fossa, and right hilum. Bronchoscopy demonstrated no focal endobronchial lesions, and cytology, Gram staining and culture of bronchoalveolar lavage fluid were non-diagnostic. Endobronchial ultrasound-guided transbronchial needle aspiration of the paratracheal and subcarinal lymph nodes using a 22-gauge transbronchial needle aspiration needle (NA-201SX-4022, Olympus, Tokyo, Japan) with five passes was performed. Pathological examination revealed fibrous tissue with abundant plasma cells. Immunohistochemical staining for immunoglobulin light chains was equivocal.

\section{QUESTION}

What is the likely diagnosis and how should this be confirmed? See page 187 for answer.

\section{Y H Khor, ${ }^{1}$ D P Steinfort, ${ }^{1}$ M R Buchanan, ${ }^{2}$ D Gunawardana, ${ }^{3}$ P Antippa, ${ }^{4}$ L B Irving, ${ }^{1}$}

1 Department of Respiratory Medicine, Royal Melbourne Hospital, Victoria, Australia; 2Department of Pathology, Royal Melbourne Hospital, Victoria, Australia; ${ }^{3}$ Department of Nuclear Medicine, Royal Melbourne Hospital, Victoria, Australia; ${ }^{4}$ Department of Cardiothoracic Surgery, Royal Melbourne Hospital, Victoria, Australia
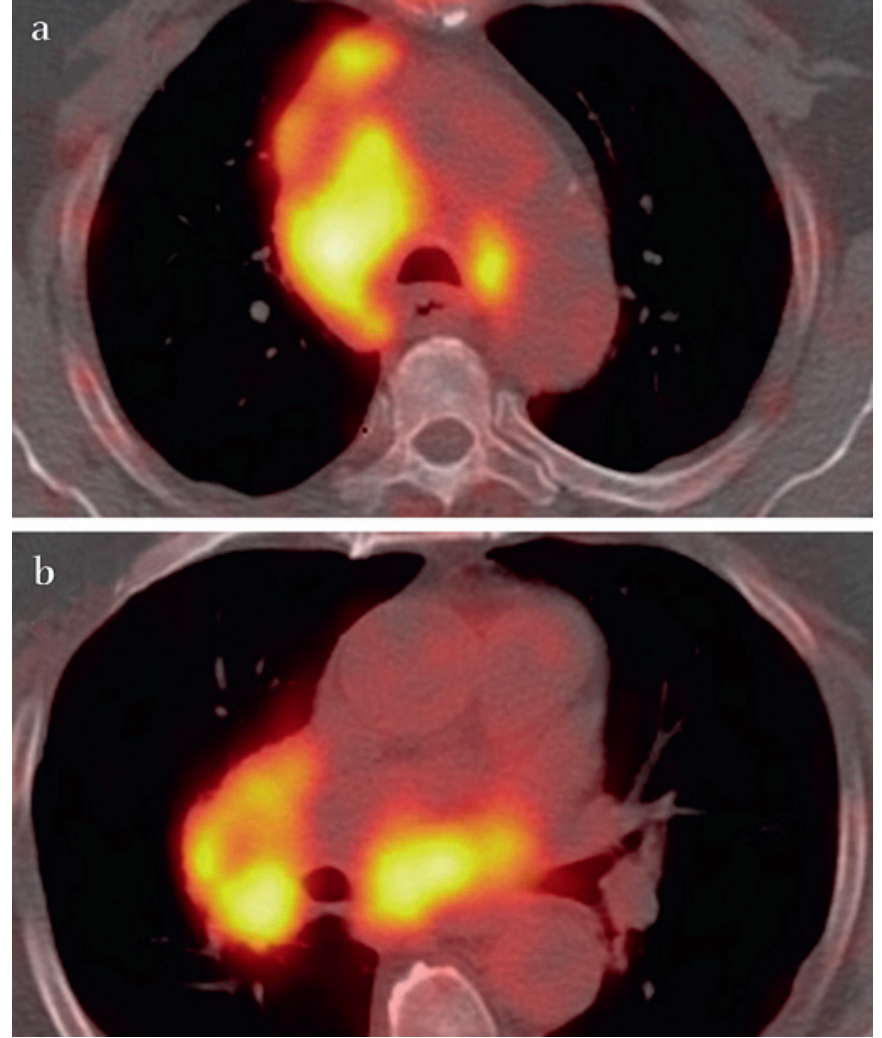

Figure 1 Positron emission tomography image demonstrating large intensely fluorodeoxyglucose (FDG)-avid masses in (a) pretracheal and (b) subcarinal and right hilar regions.

Correspondence to Dr Yet Hong Khor, Department of Respiratory Medicine, Royal Melbourne Hospital, Grattan Street, Parkville, Victoria 3050, Australia; YetHong.Khor@mh.org.au

\section{Competing interests None.}

Patient consent Obtained.

Provenance and peer review Not commissioned; externally peer reviewed. Thorax 2010;65:138. doi:10.1136/thx.2009.123828 